\title{
"A Debate: Is Surgical Intervention for Cystic Neoplasms of the Pancreas Being Overutilized"
}

\author{
Keith D. Lillemoe
}

Received: 21 August 2013 / Accepted: 15 October 2013 /Published online: 29 October 2013

(C) 2013 The Society for Surgery of the Alimentary Tract

Keywords Pancreatic cystic $\cdot$ Intraductal papillary mucinous neoplasms $\cdot$ Pancreatic cancer

At the 2012 meeting of the Society for Surgery of the Alimentary Tract, an interesting and stimulating debate was held on the topic of the appropriate utilization of surgical resection for cystic neoplasms of the pancreas. The view that surgical resection is underutilized was supported by Dr. Max Schmidt from Indiana University with the opposing view that surgical resection is overutilized argued by Dr. Peter Allen of the Memorial-Sloan Kettering Cancer Center. Both speakers are recognized internationally as outstanding pancreatic surgeons who have published extensively on the topic of cystic neoplasms of the pancreas and specifically sidebranch intraductal papillary mucinous neoplasms (IPMNs), which is certainly the main issue of importance in both this debate and in the clinical management of patients. Furthermore, both authors are among the leaders nationally in establishing prospective databases to follow patients with cystic neoplasms at their own institutions. The authors have summarized their opinions in the following manuscripts and have provided evidence to support their beliefs.

Despite the eloquence of the speakers, the debate as to the optimal management of small, asymptomatic side-branch IPMNs remains unresolved. In fact, two recent publications have added fuel to the fire. First, an updated set of recommendations have been put forth by the international consensus conference working group. ${ }^{1}$ These recommendations have updated the original "Sendai guidelines" originally published in $2007 .^{2}$ These new guidelines appear to, among other points, provide recommendations further "swinging the pendulum" towards observation of "low-risk" side-branch

K. D. Lillemoe $(\bowtie)$

Department of Surgery, The Massachusetts General Hospital,

Boston, MA, USA

e-mail: klillemoe@partners.org
IPMNs. On the other hand, a recent, somewhat controversial paper from the surgical group in Heidelberg, Germany, has defined a $24.6 \%$ incidence of malignancy in "Sendai-negative" branch-duct IPMNs with $15.9 \%$ of all such tumors harboring invasive cancer. ${ }^{3}$ Interestingly, the incidence of malignancy in the Heidelberg report is nearly identical to the reported incidence of cancer in a series of resected highly selected branch-duct IPMNs published by these two debaters. ${ }^{4,5}$

This debate will obviously continue and may intensify as surgeons and gastroenterologists care for an ever increasing number of these patients with pancreatic cystic neoplasms, often as incidental findings. The proper balance of early diagnosis and/or prevention of life-threatening pancreatic cancer versus both short-term and long-term morbidity and rare mortality of major pancreatic resection is clearly yet to be defined. Also of significance in this time of increased emphasis on the cost of healthcare are questions related to both the "appropriateness" and cost-effectiveness of major pancreatic surgical procedures versus long-term observation with serial expensive imaging for decades of life. Clearly the debate at the SSAT has just scratched the surface of these issues and will ultimately continue on into the future.

\section{References}

1. Tanaka M, Fernandez-del Castillo C, Adsay V, et al: International consensus guidelines 20120 for the management of IPMN and MCN of the pancreas. Pancreatology 2012; 12:183-97.

2. Tanaka M, Chari S, Adsay V, et al: International consensus guidelines for management of intraductal papillary mucinous neoplasms and mucinous cystic neoplasms of the pancreas. Pancreatology 2006; 6:17-32.

3. Fritz S, Klaus V, Bergmann F et al. Small (Sendai negative) branchduct IPMNs: Not harmless. Ann Surg 2012; 256:313-320.

4. Schmidt CM, White PB, Waters JA, et al. Intraductal papillary mucinous neoplasms: Predictors of malignant and invasive pathology. Ann Surg 2007; 246:644-654.

5. Allen PJ, D'Angelica AM, Gomen M, et al. A Selective approach to the resection of cystic lesions of the pancreas: Results from 539 consecutive patients. Ann Surg 2006; 244:572-582. 